\title{
Communication Strategies
}

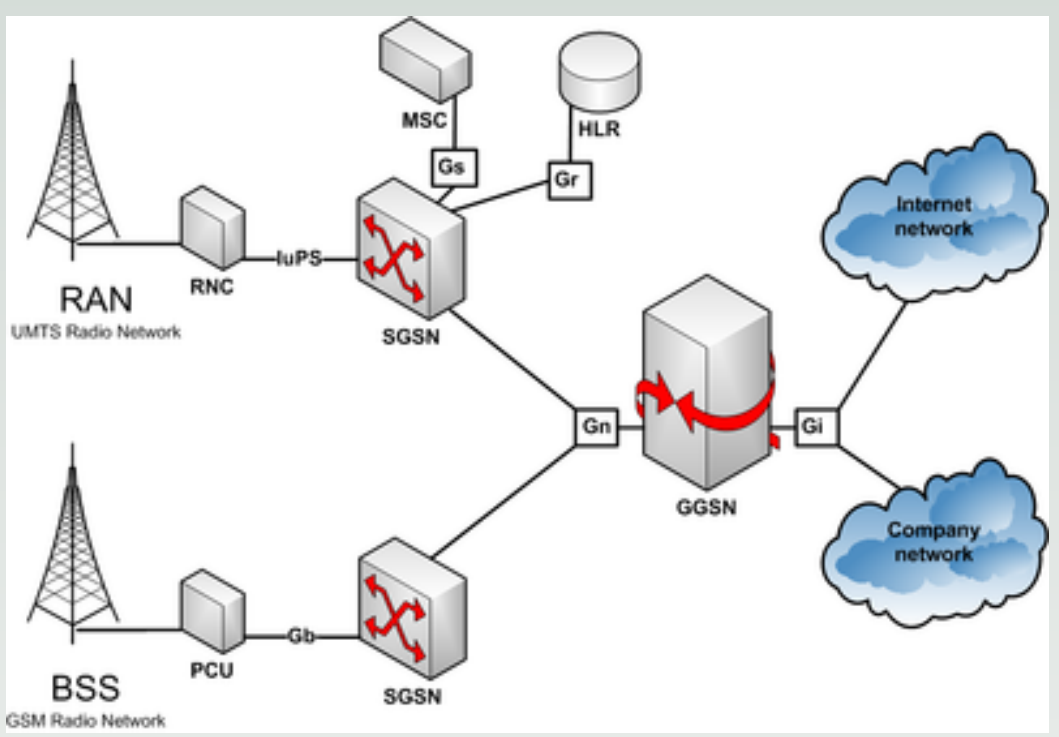




\section{This is not a real-time system}

- SPMs do not rely on second-by-second polling

- SPMs do not require an "Always-On" connection

- The bandwidth requirements are low, and the files come in bursts rather than a constant stream

- We have options available that we do not have with a real-time TMS

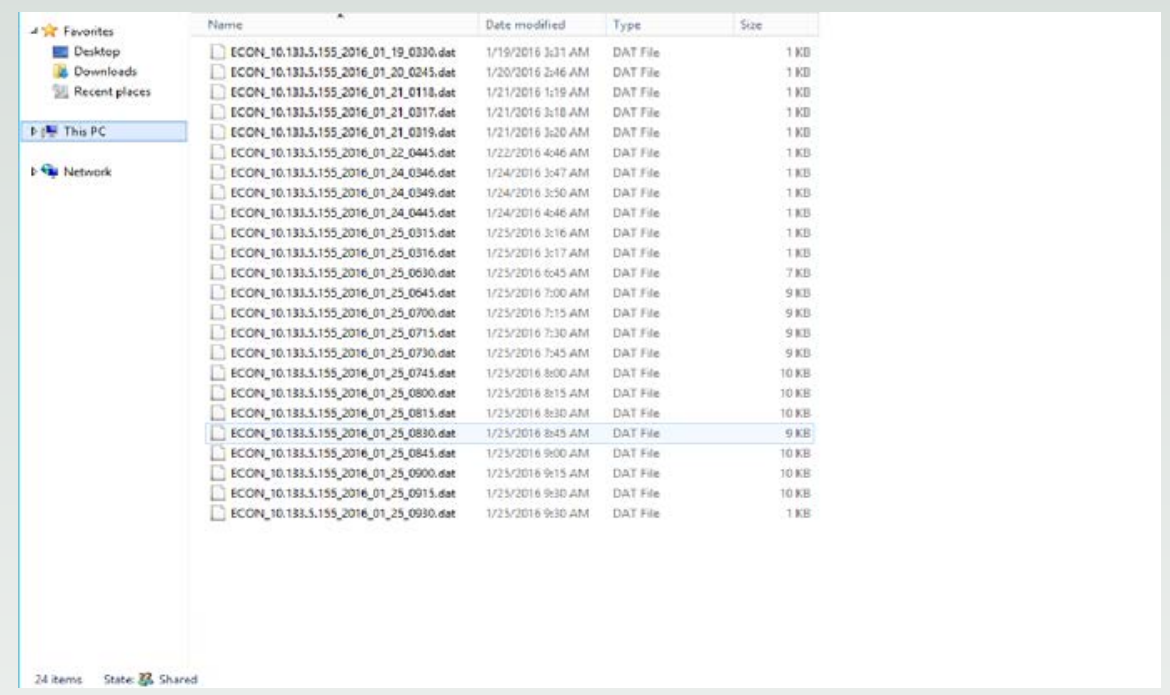




\section{The Goal}

Establish an FTP or web client connection with each controller at least once a day. 


\section{Some Terms}

Bandwidth: The amount of bits that can be transmitted of a given time. Typically measured in kbps (KiloBITS per second). This is different than KiloBYTES per second by an order of magnitude

Latency: The time it takes for a packet to travel from the sending node to the receiving node. Typically measured in milliseconds. For Ethernet, latency is highly variable

IP Address: Internet Protocol Address. A unique identifier assigned to each device on a network that describes where on the network the device is. Usually looks like 192.168.127.10

FTP: File Transfer Protocol. It is used to reliably transmit files over IP based networks. Very popular, but has some security risks 


\section{FTP Security Risks}

All transmissions are in clear text

(Unless you use the special FTPS, which no one does)

"Transmissions" includes usernames and password

Most controller vendors only have root level accounts set up

This means that anyone who is watching the wire can get complete control of almost every controller on the network 


\section{The Options}

Fiber Optic Cable (The Obvious Choice)

- If you already have it, it is wonderful

- Provides huge amounts of bandwidth and flexibility

- Installing it requires trenching and it can be expensive 


\section{The Options}

Twisted Wire Pair

- If you already have it, consider installing DSL equipment

- DSL will provide sufficient bandwidth for the SPMs, with plenty to spare

- Maintenance is relatively straight forward

- If you don't already have it, don't install it. Most of the costs are in trenching, so you may as well put in fiber 


\section{The Options}

Dial up (Closed loop)

- Dial up works fine for SPMs

- You must be able to pass IP over the modems

- You must have IP/Ethernet connections between the cabinet in the loop 


\section{The Options}

Wireless radio

- Only Ethernet radios will work

- Radios require line-of-sight. Tree, buildings and other objects will interfere

- Less reliable than wired solutions, but do not require trenching 


\section{The Options}

Cable/Telephone Providers

- Most providers already have coverage around intersections

- Providers often either charge a flat monthly fee, or charge by how much bandwidth gets used (or both) 


\section{The Options}

Cell modems

- Cell modems use the cellular phone network

- There is usually a month fee AND a bandwidth charge that comes with cell modems

- They can be deployed any place there is cell coverage 


\section{The Options}

Satellite

- Usable almost anywhere there is a clear view of the sky

- When compared with other subscription services, this on is expensive 


\section{Putting it all Together}

- Don't be afraid to mix and match technologies to get the comm you need.

- Always try to leverage what you have.

- SPMs do not (so far) interfere with central signal systems. As much as possible, design a network that meets both needs. 


\section{Questions?}

